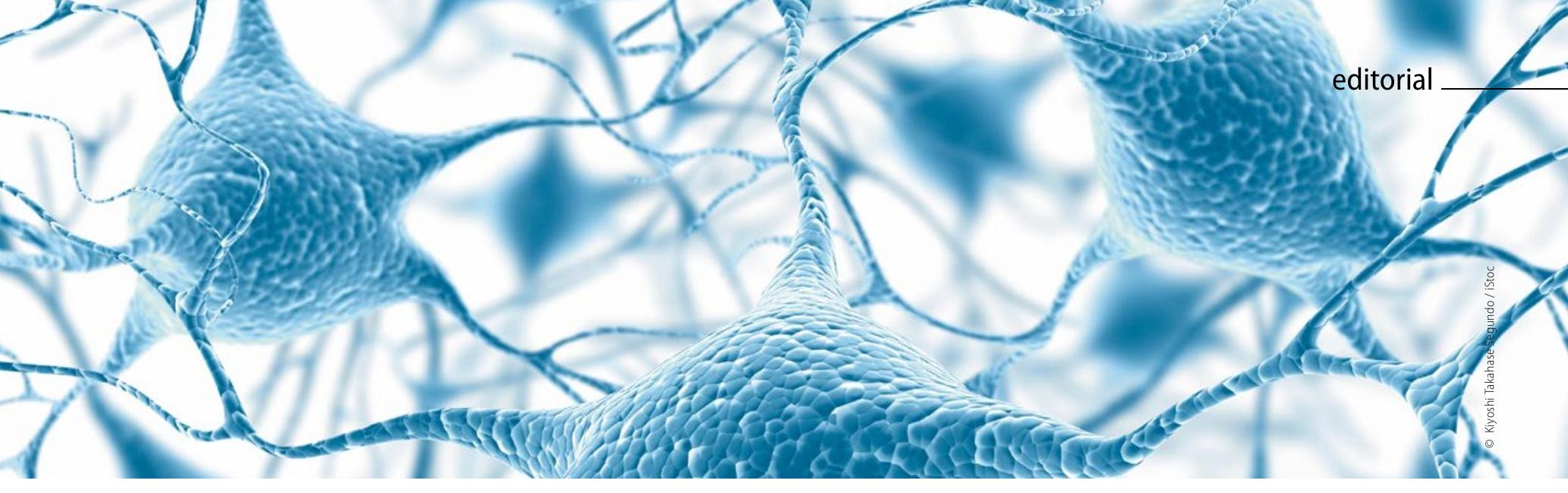

\title{
Research Domain Criteria - in Deutschland noch zu wenig bekannt
}

Es war bereits im Jahr 2008, als Thomas Insel und die National Institutes of Mental Health in den USA mit der strukturierten Definition und Erforschung der sogenannten Research Domain Criteria begannen. Ausgangspunkt war die eigentlich schon uralte Beobachtung, dass es sich bei den nach der ICD-10 beziehungsweise dem DSM-5 gestellten Diagnosen um diagnostische Übereinkünfte von Experten handelt und nicht etwa um Krankheitsentitäten, denen gleiche neurobiologische oder psychologische Ursachen zugrunde liegen. Mit den Research Domain Criteria wird versucht, umschriebene Dysfunktionalitäten zu erfassen, von denen man annehmen kann, dass ihnen eine umschriebene neurobiologische Ursache zugrunde liegt, die sich besser erforschen lässt, als wenn man die Ursachen von Krankheiten, wie sie im ICD-10 definiert sind, erforschen möchte.

Ein Beispiel ist die "gestörte Impulskontrolle“, die sich einerseits mittels neuropsychologischer Testverfahren gut operationalisieren lässt und der andererseits wahrscheinlich die Störung eines oder mehrerer umschriebener neurobiologischer Regelkreise zugrunde liegt. Gestörte Impulskontrolle ist damit nur eine Dimension einer Störung, die bei unterschiedlichen Krankheitsdiagnosen wie etwa ADHS, bipolaren Störungen, Borderline-Persönlichkeitsstörungen oder der antisozialen Persönlichkeitsstörung auftreten kann. Basierend auf dem Ansatz der Research Domain Criteria würde man nun nicht wie üblicherweise etwa die Neurobiologie der Borderline-Persönlichkeitsstörung, sondern die Neurobiologie gestörter Impulskontrolle studieren, indem man Personen mit unterschiedlichen ICD-10- oder DSM5-Diagnosen in einem Untersuchungsdesign zusammenbringt, wenn sie das Symptom einer (neuropsychologisch operationalisierten) Impulskontrollstörung aufweisen. Das erhöht die Wahrscheinlichkeit, der neurobiologischen Regelkreisstörung näher zu kommen, da man studieren kann, wie die neurobiologisch gleiche Regelkreisstörung aussieht, wenn deren unterschiedliche Ausprägung bei verschiedenen Erkrankungen miteinander verglichen und in Beziehung gesetzt wird. Ähnlich würde man auch bei der Medikamentenentwicklung vorgehen: Ziel wäre nicht die Entwicklung eines Medikamentes etwa gegen bipolare Störungen, sondern beispielsweise gegen eine gestörte Impulskontrolle. Hier würden dann in ein randomisiert kontrolliertes Studiendesign Patienten mit unterschiedlichen

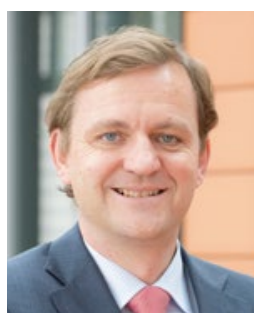

Univ.-Prof. Dr. med. Klaus Lieb

Direktor der Klinik für Psychiatrie und

Psychotherapie, Universitätsklinikum Mainz

E-Mail: klaus.lieb@unimedizin-mainz.de

Diagnosen eingeschlossen, die als Kernsymptomatik eine gestörte Impulskontrolle aufweisen.

Dass der Vollzug eines solchen oder ähnlichen Paradigmenwechsels in der psychiatrischen Forschung dringend geboten wäre, liegt auf der Hand: Die psychiatrische Medikamentenentwicklung befindet sich seit mindestens 20 Jahren in einer katastrophalen Innovationskrise und trotz hoch entwickelter neurobiologischer Forschungsmethoden etwa in der Genetik oder Bildgebung sind wir den neurobiologischen Ursachen psychiatrischer Erkrankungen keinen großen Schritt näher gekommen. Die konsequente Umsetzung des RDCs-Ansatzes wäre ein Schritt in die richtige Richtung. Das NIMH fördert neurobiologische Forschung konsequenterweise nur noch dann, wenn sie von kategorialen Krankheitsdiagnosen weggeht. Das müsste auch die DFG in Deutschland tun.

Warum ein Umdenken so schwierig ist, liegt an nachvollziehbaren Gründen: Diagnosen als diagnostische Übereinkünfte helfen Ärzten und Patienten, Krankheitssymptome einzuordnen, fassbar und verständlich zu machen und sind die zugrunde liegende Logik für das kassenärztliche Abrechnungswesen und die Krankenkassen. Für Diagnosen sind Chefärzte Experten, nach ihnen richten sie ihre Karrieren aus, Medikamente werden für Diagnosen entwickelt und verkauft. Ein Paradigmenwechsel wäre also eine riesige Kraftanstrengung - kein Wunder, dass es trotz anderslautender Ankündigungen mit dem DSM-5 nur ansatzweise gelungen ist, das Ruder herumzureißen - aber die Kraftanstrengung würde sich lohnen, wenn wir nicht in 20 Jahren immer noch mit der gleichen kleinen Handvoll von Wirkprinzipien von Psychopharmaka dastehen wollen.

Klaus Lieb, Mainz 